\title{
Obtainment of the density of states in the band tails of hydrogenated amorphous silicon
}

Leonardo Kopprio, Christophe Longeaud, and Javier Schmidt

Citation: Journal of Applied Physics 122, 085702 (2017); doi: 10.1063/1.4999626

View online: http://dx.doi.org/10.1063/1.4999626

View Table of Contents: http://aip.scitation.org/toc/jap/122/8

Published by the American Institute of Physics

\section{Articles you may be interested in}

Nanostructure evolution of magnetron sputtered hydrogenated silicon thin films Journal of Applied Physics 122, 075302 (2017); 10.1063/1.4998455

Effects of a chirped bias voltage on ion energy distributions in inductively coupled plasma reactors Journal of Applied Physics 122, 083301 (2017); 10.1063/1.4993785

Enhanced optical, electrical, and mechanical characteristics of $\mathrm{ZnO} / \mathrm{Ag}$ grids/ZnO flexible transparent electrodes Journal of Applied Physics 122, 085501 (2017); 10.1063/1.4994965

Current-matching versus non-current-matching in long wavelength interband cascade infrared photodetectors Journal of Applied Physics 122, 083102 (2017); 10.1063/1.4989382

Electronic band structures and excitonic properties of delafossites: A GW-BSE study Journal of Applied Physics 122, 085104 (2017); 10.1063/1.4991913

Structural vs. compositional disorder in thermal conductivity reduction of SiGe alloys Journal of Applied Physics 122, 045104 (2017); 10.1063/1.4994169

\section{AlP | Journal of}

Save your money for your research.

It's now FREE to publish with us no page, color or publication charges apply.

\section{Publish your research in the} Journal of Applied Physics to claim your place in applied physics history. 


\title{
Obtainment of the density of states in the band tails of hydrogenated amorphous silicon
}

\author{
Leonardo Kopprio, ${ }^{1}$ Christophe Longeaud, ${ }^{2}$ and Javier Schmidt ${ }^{1,3}$ \\ ${ }^{1}$ Instituto de Física del Litoral (IFIS-Litoral), CONICET-UNL, Güemes 3450, S3000GLN Santa Fe, Argentina \\ ${ }^{2}$ GEEPS, CNRS, CentraleSupelec, UPSud, UPMC, 11 Rue Joliot Curie, 91190 Gif sur Yvette, France \\ ${ }^{3}$ Facultad de Ingeniería Química, Universidad Nacional del Litoral, Santiago del Estero 2829 , \\ S3000AOM Santa Fe, Argentina
}

(Received 4 May 2017; accepted 9 August 2017; published online 25 August 2017)

\begin{abstract}
In this work, we present two new pairs of formulas to obtain a spectroscopy of the density of states (DOS) in each band tail of hydrogenated amorphous silicon (a-Si:H) from photoconductivity-based measurements. The formulas are based on the knowledge of the small-signal recombination lifetime $\tau^{\prime}$, the characteristic decay time of the concentration of trapped carriers generated in excess by the illumination, and that can be measured by methods like the Oscillating Photocarrier Grating (OPG) or Moving Grating Technique (MGT). First, we deduce the formulas and test their accuracy by numerical simulations using typical a-Si:H parameters. Next, we characterize an a-Si:H sample using well-known methods, like Fourier transform photocurrent spectroscopy to evaluate the valence band tail and modulated photoconductivity to measure the conduction band tail. We also performed measurements of steady-state photoconductivity, steady-state photocurrent grating and MGT, for a range of generation rates. From these measurements-and taking typical values for the capture coefficients, the extended states mobilities and the DOS at the band edges-we apply the new formulas to get the band tails. We find that the results obtained from the application of our formulas are in good agreement with those found with the traditional methods for both band tails. Moreover, we show that MGT/OPG measurement to get $\tau^{\prime}$ can be avoided if one of the band tails is measured by one of the traditional methods, since the known band tail can be used to evaluate $\tau^{\prime}$ with one pair of equations, and then the other pair can be applied to get the other band tail. Published by AIP Publishing.
\end{abstract}

[http://dx.doi.org/10.1063/1.4999626]

\section{INTRODUCTION}

The disorder present in the structure of hydrogenated amorphous Silicon (a-Si:H) leads to the presence of localized states that extend inside the gap of the semiconductor as band tails. On the other hand, defect states located around the midgap originate from coordination defects. Both shallow states of the band tails and deep states associated with dangling bonds have a decisive influence on the transport properties of the material, as these states control processes of recombination, capture, and re-emission of carriers.

The determination of the density of states (DOS) in the gap of a-Si: $\mathrm{H}$ is a subject of interest not only from the point of view of basic science, but also due to the many technological applications of a-Si:H. To estimate the DOS between the valence band edge and the Fermi level, some methods based on the deconvolution of the absorption coefficient have been proposed, ${ }^{1-3}$ which in turn can be obtained by techniques like Constant Photocurrent Method (CPM), ${ }^{4}$ Dual Beam Photoconductivity (DBP), ${ }^{5}$ Photothermal Deflection Spectroscopy (PDS) ${ }^{6}$ or Fourier Transform Photocurrent Spectroscopy (FTPS). ${ }^{7}$ On the other hand, methods based on the photoconductivity of intrinsic a-Si:H generally allow to obtain a part of the DOS between the Fermi level and the conduction band edge. Among these, the method of modulated photoconductivity (MPC) is one of the most widely used ${ }^{8,9}$ Some methods based on making interference between two coherent light beams have also been proposed for this part of the DOS of a-Si:H; ${ }^{10}$ in particular, we have recently proposed the combined use of Oscillating Photocarrier Grating (OPG) and Moving Grating Technique (MGT) for this purpose. ${ }^{11}$ These methods provide information about the small-signal excess electron lifetime, $\tau_{n}{ }^{\prime}$, which is the characteristic time with which the total electron concentration adapts to a small change in the illumination intensity. In recent publications, we have proposed a simple and a more sophisticated formula for assessing the DOS in the conduction band tail (CBT) from measurements of OPG/MGT. ${ }^{12,13}$

For a semiconductor under a spatially and temporally uniform generation rate $G$ (steady state conditions), the total concentration of electrons $n_{T}$ should be equal to the total concentration of holes $p_{T}$, as can be proved from Maxwell's first equation. Consequently, the partial derivatives with respect to the generation rate should also be equal: $\tau_{n}{ }^{\prime}=\partial n_{T} /$ $\partial G$ should be equal to the corresponding time for holes, $\tau_{p}{ }^{\prime}=\partial p_{T} / \partial G$. Therefore, this common small-signal recombination lifetime $\tau^{\prime}$ should provide information not only about the CBT but also about the valence band tail (VBT). In this paper we show that this is indeed the case, and that a spectroscopy of the VBT is possible from measurements of OPG/MGT combined with measurements of steady state 
photoconductivity (SSPC) and steady-state photocarrier grating (SSPG). Moreover, we provide a formula for the obtainment of the conduction band tail that uses the same measurements and is more accurate than simpler formulas found in the literature. ${ }^{11,13}$

This work is organized as follows. In Sec. II A, we present the new equations, describing how they should be implemented and their range of validity. The formal derivation of these equations is presented in Sec. II B, and it is not needed for the understanding of the rest of the paper. In Sec. III, we test the deduced equations by means of numerical calculations using typical parameters for undoped a-Si:H, comparing these results with the initially introduced DOS. In Sec. IV, the experimental equipment and procedures are described, and the results of our measurements are presented and discussed in Sec. V. The conclusions are summarized in Sec. VI. Finally, the Appendix gathers some basic equations valid for amorphous semiconductors that are used in the rest of this work.

\section{THEORETICAL DEVELOPMENTS}

\section{A. New equations for band tail spectroscopy}

For an amorphous semiconductor like a-Si:H, naturally $n$-type when not intentionally doped, previous studies have reported on the possibility to obtain the density of states, $N(E)$, in the conduction band tail at the quasi-Fermi level for trapped electrons, $E_{t n}$, from measurements of the smallsignal lifetime $\tau^{\prime}$ and the steady-state photoconductivity $\sigma$, using the formula ${ }^{11,13}$

$$
N^{C B T}\left(E_{t n}\right) \cong \frac{\tau^{\prime} G}{k_{b} T \gamma_{\sigma}} \frac{\sin \left(\pi T / T_{C}\right)}{\pi T / T_{C}},
$$

where $G$ is the steady-state generation function, $k_{b}$ is the Boltzmann's constant, $T$ is the absolute temperature, $T_{C}$ is the characteristic temperature of the conduction band tail, and $\gamma_{\sigma}$ is the exponent describing the power-law dependence of photoconductivity with generation rate

$$
\sigma \propto G^{\gamma_{\sigma}} .
$$

In turn, $\tau^{\prime}$ can be obtained from the photoconductivity-based techniques MGT/OPG. ${ }^{12}$ To calculate $E_{t n}$, the steady-state photoconductivity $\sigma$ measured under an illumination that produces a uniform generation of carriers within the material, should be used in the following formula:

$$
E_{t n} \cong E_{C}-k_{b} T \ln \left(\frac{q \mu_{n} N_{C}}{\sigma}\right) .
$$

$E_{C}$ is the energy of the conduction band edge (equal to the mobility gap if we take the zero of energy at the valence band edge), $\mu_{n}$ is the free electron mobility, and $N_{C}$ is the effective density of states at the conduction band edge. This equation can be deduced from Eq. (A19) by neglecting the hole contribution, an approximation valid for undoped or $n$ type a-Si:H. For this case, the quasi Fermi levels for trapped and free electrons are almost identical.

Ventosinos et al. ${ }^{11}$ deduced a simpler version of Eq. (1) for the first time, while Longeaud et al. ${ }^{14}$ proposed the actual Eq. (1) as a way of gaining precision. Performing measurements of $\sigma, \gamma_{\sigma}$, and $\tau^{\prime}$ for different temperatures and generation rates, an estimation of the DOS in the conduction band tail of a-Si:H can be obtained from Eqs. (1)-(3).

In this work, we present two new pairs of formulas (deduced in Sec. II B) applicable to amorphous photoconductors verifying the inequality $\mu_{n} n_{0} \gg \mu_{p} p_{0}$, where $n_{0}$ and $p_{O}$ are the concentrations of free electrons and holes under illumination, and $\mu_{p}$ is the extended-state hole mobility. The DOS in the conduction band tail can be obtained from

$$
N^{C B T}\left(E_{t n}\right) \cong \frac{q \mu_{n} \sin \left(\frac{\pi T}{T_{C}}\right)\left(\frac{R_{C} \sigma}{q \mu_{n}}+\frac{G L_{a m b}^{2}}{2 k_{b} T \mu_{p}}\right)^{2}\left(\tau^{\prime}-\frac{\gamma_{\sigma} \sigma}{q \mu_{n} G}\right)}{R_{C} \pi \sigma\left(\gamma_{\sigma}\left(\frac{L_{a m b}^{2}}{2 \mu_{p}}+\frac{R_{C} \sigma k_{b} T^{2}}{q \mu_{n} G T_{C}}\right)-\frac{L_{a m b}^{2}}{\mu_{p}}\left(\frac{1}{2}+\gamma_{L}\right)\left(1-\frac{T}{T_{C}}\right)\right)}
$$

$$
E_{t n} \cong E_{C}+k_{b} T \ln \left(\frac{\frac{R_{C} \sigma}{q \mu_{n}}+\frac{G L_{a m b}^{2}}{2 k_{b} T \mu_{p}}}{R_{C} N_{C}}\right),
$$

where $R_{C}=\frac{c_{n}^{C B T}}{c_{p}^{C B T}}$ is the ratio of the capture coefficients for electrons and holes in the conduction band tail and $L_{a m b}$ is the ambipolar diffusion length that can be measured with the SSPG technique. ${ }^{15}$ In Eq. (4) also appears the exponent $\gamma_{L}$ of the power-law relationship between $L_{a m b}$ and $G$

$$
L_{a m b} \propto G^{\gamma_{L}} .
$$

Therefore, to use Eqs. (4) and (5) we need an estimate of $R_{C}$, and the extended-state mobilities $\mu_{n}$ and $\mu_{p}$.

The parameters $\gamma_{\sigma}$ and $\gamma_{L}$, defined in Eqs. (2) and (6), can be related to the more fundamental parameters $\gamma_{n}$ and $\gamma_{p}$ defined by the equations $n_{0} \propto G^{\gamma_{n}}$ and $p_{0} \propto G^{\gamma_{p}}$. Indeed, for an undoped or n-type semiconductor a simple calculation shows that $\gamma_{\sigma} \cong \gamma_{n}$ and $\gamma_{L} \cong\left(\gamma_{p}-1\right) / 2$. From this last expression we can see that, since $\gamma_{p}$ is usually less than $1, \gamma_{L}$ should be negative.

The DOS in the valence band tail can be obtained from 


$$
N^{V B T}\left(E_{t p}\right) \cong \frac{2 \mu_{p}\left(\frac{R_{V} \sigma}{\mu_{n} q}+\frac{G L_{a m b}^{2}}{2 k_{b} T \mu_{p}}\right)^{2} \sin \left(\frac{\pi T}{T_{V}}\right)\left(\tau^{\prime}-\left(\frac{1}{2}+\gamma_{L}\right) \frac{L_{a m b}^{2}}{k_{b} T \mu_{p}}\right)}{\pi L_{a m b}^{2}\left(\frac{R_{V} \gamma_{\sigma} \sigma}{q \mu_{n}}\left(\frac{T}{T_{V}}-1\right)+\left(1+2 \gamma_{L}\right)\left(\frac{G L_{a m b}^{2}}{2 k_{b} T_{V} \mu_{p}}+\frac{R_{V} \sigma}{q \mu_{n}}\right)\right)},
$$

$$
E_{t p} \cong E_{V}-k_{b} T \ln \left(\frac{\frac{R_{V} \sigma}{q \mu_{n}}+\frac{G L_{a m b}^{2}}{2 \mu_{p} k_{b} T}}{N_{V}}\right),
$$

where $E_{V}$ is the energy of the valence band edge, $R_{V}=\frac{c_{n}^{V B T}}{c_{p}^{V B T}}$ is the ratio of the capture coefficients for electrons and holes in the valence band tail (VBT), $E_{t p}$ is the quasi-Fermi level for trapped holes, $T_{V}$ is the characteristic temperature of the valence band tail, and $N_{V}$ is the effective density of states at the valence band edge. With the exception of $N_{V}$ and $R_{V}$, Eqs. (7) and (8) include the same parameters as Eqs. (4) and (5); therefore, if the parameters have been measured to obtain one of the band tails, it would be possible to get the other band tail without performing almost any additional measurement.

The implementation of DOS spectroscopy from Eqs. (4) and (5), or from Eqs. (7) and (8), is very similar to the procedure proposed by Longeaud et al. ${ }^{14}$ using Eqs. (1) and (3). However, two extra parameters need to be measured, $L_{a m b}$ and $\gamma_{L}$, for the same temperatures and generation rates that were used to measure the other common parameters: $\sigma, \gamma_{\sigma}$, and $\tau^{\prime}$. The temperature should be low enough and the generation rate high enough for the quasi-Fermi levels to fall in the respective band tail regions of the DOS. Equations (4) and (5) have a more stringent temperature limitation than Eqs. (7) and (8), because in a-Si:H $T_{C}<T_{V}$. The smaller the $T_{C}$, the smaller is the high-temperature limit for the applicability of Eq. (4), since we should approximately have $T \leq$ $0.75 T_{C}$. For example, for a highest-quality material having $T_{C} \sim 220 \mathrm{~K},{ }^{16}$ the temperature should not be higher than $165 \mathrm{~K}$ to guaranty the applicability of Eqs. (4) and (5). The origin of this limitation will be explained in Sec. II B.

To obtain $\tau^{\prime}$ from OPG/MGT, it is necessary that the conditions of the experiment situate the sample in the lifetime regime for a sufficiently large range of illuminations and/or temperatures, ${ }^{1-14}$ which cannot be achieved for certain a-Si:H samples. In that case, it is difficult to get $\tau^{\prime}$ from OPG/MGT with good precision, and thus Eqs. (4) or (7) could not be directly applied to determine the band tails. Nevertheless, those equations are still useful if one of the band tails is previously measured with another technique. For example, if we have measured the VBT with one of the methods of Refs. 1-3, we can rewrite Eq. (7) for the evaluation of $\tau^{\prime}$ as a function of $N^{V B T}\left(E_{t p}\right)$. This equation, in combination with Eq. (8), can be used for the obtention of $\tau^{\prime}$ through the measurement of $\sigma, \gamma_{\sigma}, L_{a m b}$, and $\gamma_{L}$ for the temperatures and generation rates that give an $E_{t p}$ falling in the VBT region. These measured parameters and the calculated $\tau^{\prime}$ can be replaced into Eqs. (4) and (5) for the obtention of the CBT. Conversely, if the CBT is first measured, for example, with the MPC technique, Eq. (4) can be solved for the evaluation of $\tau^{\prime}$ as a function of $N^{C B T}\left(E_{t n}\right)$. The known value of $N^{C B T}$, used in combination with Eq. (5) and through the measurement of $\sigma, \gamma_{\sigma}, L_{a m b}$, and $\gamma_{L}$, can be used for the obtention of $\tau^{\prime}$ in the low temperature and high generation rate regime. The measured parameters with the calculated $\tau^{\prime}$ can be replaced into Eqs. (7) and (8) to get the VBT.

The effective DOSs at the band edges [Eqs. (A20) and (A21)], required to obtain the quasi-Fermi levels, depend on the precise energy shape of the extended-states bands. For simplicity in this work we assume a uniform density of states in the bands beyond the mobility edges, ${ }^{16}$ but a more precise treatment can be implemented following Refs. 17 and 18. The equations presented in this section are valid for intrinsic and $n$-type a-Si:H, and in these cases the ones in the denominators of Eqs. (A20) and (A21) can be neglected, since in $n$ doped a-Si:H the activation energy is always greater than $0.15 \mathrm{eV} .{ }^{16}$ With these approximations we get for the effective density of states $N_{C, V}=k_{B} T N\left(E_{C, V}\right)$, which is the expression that we have used in this work.

It is important to mention that the new pairs of formulas presented above, as well as the formula presented by Longeaud et al., ${ }^{14}$ have to be used recursively, since the band tails' characteristic temperatures and the DOS at the band edges are present in the expressions. So, it is necessary to have an initial estimate of these two parameters, which are then refined by the recursive application of the formulas.

\section{B. Deduction of the formulas}

The definition of the small-signal recombination lifetime is

$$
\tau^{\prime}=\frac{\partial n_{T}}{\partial G}=\frac{\partial n_{0}}{\partial G}+\frac{\partial n_{t}}{\partial G}=\frac{\partial p_{T}}{\partial G}=\frac{\partial p_{0}}{\partial G}+\frac{\partial p_{t}}{\partial G},
$$

where $n_{T}$ and $p_{T}$ are the total (free + trapped) concentrations of electrons and holes, $n_{0}$ and $p_{0}$ are the concentrations of free electrons and holes, and $n_{t}$ and $p_{t}$ are the concentrations of trapped electrons and holes. The reader should not confuse $\tau^{\prime}$ with the full-signal recombination lifetime $\tau_{R}$, defined in Eq. (A22), characterizing the decay time of the total carrier concentration when the illumination is suddenly suppressed. Replacing Eq. (A16) in expression (9), we obtain

$$
\tau^{\prime}=\frac{\partial n_{0}}{\partial G}+\int_{E_{v}}^{E_{c}} \frac{\partial f_{0}}{\partial G} N^{A C C}(E) d E,
$$


where $f_{0}$ is the occupation function under steady-state illumination and $N^{A C C}(E)$ is the density of acceptor states. Note here that the CBT states are considered as acceptor states. In the acceptor region of the DOS (upper half of the gap), the occupation function can be approximated by

$$
f_{0} \approx \frac{c_{n} n_{0}}{c_{n} n_{0}+c_{p} p_{0}+e_{n}(E)}=\frac{c_{n} n_{0}}{\left(c_{n} n_{0}+c_{p} p_{0}\right)\left(1+e^{\frac{E-E_{n}}{k_{b} T}}\right)},
$$

where $e_{n}(E)$ is the electron emission coefficient. Performing the derivative inside the integral of Eq. (10) we have

$$
\begin{aligned}
\tau^{\prime} \cong & \frac{\partial n_{0}}{\partial G}+\int_{E_{v}}^{E_{c}} \frac{\partial}{\partial G}\left(\frac{1}{1+e^{\frac{E-E_{t n}}{k_{b} T}}}\right) \frac{c_{n} n_{0} N^{C B T}(E) d E}{\left(c_{n} n_{0}+c_{p} p_{0}\right)} \\
& +\int_{E_{v}}^{E_{c}} \frac{\partial}{\partial G}\left(\frac{c_{n} n_{0}}{c_{n} n_{0}+c_{p} p_{0}}\right) \frac{N^{C B T}(E) d E}{1+e^{\frac{E-E_{t n}}{k_{b} T}}} .
\end{aligned}
$$

The first integral of Eq. (12) is given by

$$
\begin{aligned}
\int_{E_{v}}^{E_{c}} & \frac{\partial}{\partial G}\left(\frac{1}{1+e^{\frac{E-E_{m}}{k_{b} T}}}\right) \frac{c_{n} n_{0} N^{C B T}(E) d E}{\left(c_{n} n_{0}+c_{p} p_{0}\right)} \\
= & \frac{c_{n} n_{0}\left(c_{n} \frac{\partial n_{0}}{\partial G}+c_{p} \frac{\partial p_{0}}{\partial G}\right)}{\left(c_{n} n_{0}+c_{p} p_{0}\right)^{2}} \int_{E_{v}}^{E_{c}} \frac{e^{\frac{E-E_{t n}}{k_{b} T}} N^{C B T}(E) d E}{\left(1+e^{\frac{E-E_{m}}{k_{b} T}}\right)^{2}} .
\end{aligned}
$$

Replacing the expression for the conduction band tail into Eq. (13), $N\left(E_{C}\right) e^{\frac{E-E_{C}}{k_{b} T_{C}}}$, and performing the substitution $x=\frac{E-E_{t n}}{k_{b} T}$ and $a=1+\frac{T}{T_{C}}$, we get

$$
\begin{aligned}
\int_{E_{v}}^{E_{c}} \frac{e^{\frac{E-E_{t n}}{k_{b} T}} N^{C B T}(E) d E}{\left(1+e^{\frac{E-E_{t n}}{k_{b} T}}\right)^{2}} & \cong k_{b} T N^{C B T}\left(E_{t n}\right) \int_{-\infty}^{+\infty} \frac{e^{a x} d x}{\left(1+e^{x}\right)^{2}} \\
& =k_{b} T N^{C B T}\left(E_{t n}\right) \frac{\pi T / T_{C}}{\sin \left(\pi T / T_{C}\right)}
\end{aligned}
$$

where the integral has been extended to $\pm \infty$ without making a large mistake due to the Dirac-delta behavior of the integrand.

The second integral of Eq. (12) can be expressed as

$$
\begin{aligned}
\int_{E_{v}}^{E_{c}} & \frac{\partial}{\partial G}\left(\frac{c_{n} n_{0}}{c_{n} n_{0}+c_{p} p_{0}}\right) \frac{N^{C B T}(E) d E}{1+e^{\frac{E-E_{t n}}{k_{b} T}}} \\
= & \frac{c_{n} c_{p}\left(p_{0} \frac{\partial n_{0}}{\partial G}-n_{0} \frac{\partial p_{0}}{\partial G}\right)}{\left(c_{n} n_{0}+c_{p} p_{0}\right)^{2}} N^{C B T}\left(E_{t n}\right) \int_{E_{v}}^{E_{c}} \frac{e^{\frac{E-E_{t n}}{k_{b} T_{c}}} d E}{1+e^{\frac{E-E_{t h}}{k_{b} T}}}
\end{aligned}
$$

Performing the substitution $x=\frac{E-E_{t n}}{k_{b} T}$ and $b=\frac{T}{T_{C}}$, and extending the limits of integration to $\pm \infty$ due to the Dirac-delta behavior of the integrand, we get

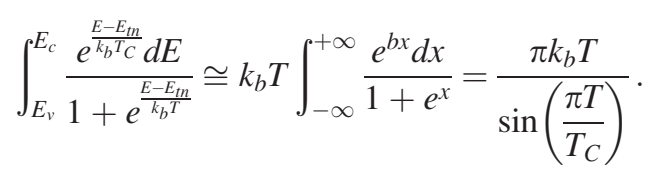

The last equalities in Eqs. (14) and (16) are strictly valid only for $T<T_{C}$, but they are actually good approximations of the first integral for temperatures $T \leq 0.75 T_{C}$. Replacing Eqs. (13)-(16) into Eq. (12), solving for $N\left(E_{t n}\right)$ and rearranging the expression, we get

$$
N^{C B T}\left(E_{t n}\right)=\frac{\sin \left(\frac{\pi T}{T_{C}}\right)\left(c_{n} n_{0}+c_{p} p_{0}\right)^{2}\left(\tau^{\prime}-\frac{\partial n_{0}}{\partial G}\right)}{c_{n} \pi k_{b} T\left(\frac{\partial n_{0}}{\partial G}\left(c_{p} p_{0}+\frac{T}{T_{C}} c_{n} n_{0}\right)-c_{p} n_{0} \frac{\partial p_{0}}{\partial G}\left(1-\frac{T}{T_{C}}\right)\right)} .
$$

Now we have to replace $n_{0}, p_{0}$, and the derivatives by parameters that can be directly measured. From Eq. (A5) we have

$$
n_{0} \cong \frac{\sigma}{q \mu_{n}}
$$

performing its derivative and using Eq. (2), we get

$$
\frac{\partial n_{0}}{\partial G} \cong \frac{\gamma_{\sigma} \sigma}{q \mu_{n} G} .
$$

Combining Eqs. (A13) and (A15), we get

$$
p_{0} \cong \frac{G L_{a m b}^{2}}{2 \mu_{p} k_{b} T},
$$

performing its derivative and using Eq. (6), we have

$$
\frac{\partial p_{0}}{\partial G} \cong\left(\frac{1}{2}+\gamma_{L}\right) \frac{L_{a m b}^{2}}{k_{b} T \mu_{p}}
$$

Replacing Eqs. (18)-(21) into Eq. (17) we get Eq. (4), while replacing Eqs. (18) and (20) into Eq. (A19) we get Eq. (5).

For the deduction of Eq. (7) we start from Eqs. (9) and (A17)

$$
\tau^{\prime}=\frac{\partial p_{0}}{\partial G}+\int_{E_{v}}^{E_{c}} \frac{\partial\left(1-f_{0}\right)}{\partial G} N^{D O N}(E) d E,
$$

where $N^{D O N}(E)$ is the density of donor states. Note that the VBT states are considered as donor states. In the donor region of the DOS (lower half of the gap), the occupation function for holes can be approximated by 


$$
1-f_{0} \cong \frac{c_{p} p_{0}}{c_{n} n_{0}+c_{p} p_{0}+e_{p}(E)}=\frac{c_{p} p_{0}}{\left(c_{n} n_{0}+c_{p} p_{0}\right)\left(1+e^{\frac{E_{t p}-E}{k_{b} T}}\right)}
$$

where $e_{p}(E)$ is the hole emission coefficient. Replacing Eq. (23) into Eq. (22) and performing the same mathematical steps that have been used to get Eq. (17), we obtain the following expression for the valence band tail:

$$
N^{V B T}\left(E_{t p}\right)=\frac{\sin \left(\frac{\pi T}{T_{V}}\right)\left(c_{n} n_{0}+c_{p} p_{0}\right)^{2}\left(\tau^{\prime}-\frac{\partial p_{0}}{\partial G}\right)}{c_{p} \pi k_{b} T\left(\frac{\partial p_{0}}{\partial G}\left(c_{n} n_{0}+\frac{T}{T_{V}} c_{p} p_{0}\right)-c_{n} p_{0} \frac{\partial n_{0}}{\partial G}\left(1-\frac{T}{T_{V}}\right)\right)} .
$$

The reader may note the symmetry of Eqs. (17) and (24) where a change of the indexes $V$ and $p$ into $C$ and $n$, respectively, transforms one equation into the other. When Eqs. (17) and (24) are numerically tested, it is found that these formulas work better for low temperatures and high generation rates, which is expected because we have assumed that the density of states is composed solely of the corresponding band tail. This approximation works better when the quasiFermi levels are far from the central region of the DOS, i.e., at low temperatures and high generation rates. We have also observed that Eq. (24) is more sensitive to errors than Eq. (17), as we will see in Secs. III and V. Replacing Eqs. (18)-(21) into Eq. (24) we get Eq. (7), while replacing Eqs. (18) and (20) into Eq. (A18) we get Eq. (8).

\section{NUMERICAL SIMULATIONS}

The accuracy in obtaining the DOS from Eqs. (4) and (5) and (7) and (8) was tested for different temperatures and light intensities by means of numerical simulations. The a$\mathrm{Si}: \mathrm{H}$ DOS used in the numerical simulations is given by the following expression:

$$
\begin{aligned}
N(E)= & N\left(E_{C}\right) e^{\frac{E-E_{C}}{k_{b} T_{C}}}+N\left(E_{V}\right) e^{\frac{E_{V}-E}{k_{b} T_{V}}}+\frac{A_{a}}{\omega_{a} \sqrt{2 \pi}} e^{\frac{-\left(E-E_{a}\right)^{2}}{2 \omega_{a}^{2}}} \\
& +\frac{A_{d}}{\omega_{d} \sqrt{2 \pi}} e^{\frac{-\left(E-E_{d}\right)^{2}}{2 \omega_{d}^{2}}}
\end{aligned}
$$

where the first term corresponds to the conduction band tail (acceptor states), the second term to the valence band tail (donor states), and the last two terms correspond to the dangling bond states, approximated by a donor and an acceptor Gaussian function. Since we concentrate on the obtainment of the DOS at the band tails, we use a simplified description of the dangling bond states based on the two states model. ${ }^{19}$ If the mobility gap of the material $E_{C}-E_{V}$ is known, the band tails are defined by their characteristic temperatures $\left(T_{C}\right.$ for the conduction band tail and $T_{V}$ for the valence band tail) and the DOS at the band edges $\left(N\left(E_{C}\right)\right.$ for the conduction band edge and $N\left(E_{V}\right)$ for the valence band edge). The distributions of deep defects are characterized by the energetic positions of the Gaussians $E_{a}$ and $E_{d}$, the standard deviations $\omega_{a}$ and $\omega_{d}$, and the total defect concentrations $A_{a}$ and $A_{d}$.
We have used a typical good quality hydrogenated amorphous silicon DOS to test the deduced equations. The values of the parameters used for the simulations are summarized in Table I.

The procedure we followed to implement Eqs. (1)-(8) is described in the following. For a given temperature, the Fermi energy $E_{f}$ is numerically found from Eq. (A1) (charge neutrality equation), and the dark concentrations of electrons and holes, $n_{d k}$ and $p_{d k}$, are obtained from Eqs. (A2) and (A3). Then, the following system of equations is numerically solved for a given generation rate $G$ to get the free electron and hole concentrations, $n_{O}$ and $p_{O}$ :

$$
\begin{gathered}
G-\int_{E_{v}}^{E_{c}} \frac{\left(c_{n} c_{p} n_{0} p_{0}-e_{p}(E) e_{n}(E)\right) N(E) d E}{c_{n} n_{0}+c_{p} p_{0}+e_{n}(E)+e_{p}(E)}=0, \\
p_{0}+\int_{E_{v}}^{E_{c}}\left[1-f_{0}(E)\right] N^{D O N}(E) d E-n_{0} \\
-\int_{E_{v}}^{E_{c}} f_{0}(E) N^{A C C}(E) d E=0 .
\end{gathered}
$$

In Eq. (26) $N(E)$ is given by Eq. (25), while in Eq. (27) unoccupied donor states are positively charged and occupied

\begin{tabular}{|c|c|}
\hline Parameters & Value \\
\hline$E_{\mathrm{V}}$ (energy at the valence band top) & 0 \\
\hline$E_{\mathrm{C}}$ (energy at the conduction band bottom) & $1.8 \mathrm{eV}$ \\
\hline$T_{\mathrm{V}}($ characteristic temperature of the VBT) & $520 \mathrm{~K}$ \\
\hline$T_{\mathrm{C}}$ (characteristic temperature of the CBT) & $350 \mathrm{~K}$ \\
\hline$N\left(E_{\mathrm{C}}\right)=N\left(E_{\mathrm{V}}\right)(\mathrm{DOS}$ at the band edges) & $10^{21} \mathrm{~cm}^{-3} \mathrm{eV}^{-1}$ \\
\hline$c_{\mathrm{n}}=c_{\mathrm{p}}($ electron and hole capture coefficient $)$ & $4 \times 10^{-9} \mathrm{~cm}^{3} \mathrm{~s}^{-1}$ \\
\hline$A_{\mathrm{d}}=A_{\mathrm{a}}$ (total density of donor and acceptor defects) & $6 \times 10^{15} \mathrm{~cm}^{-3} \mathrm{eV}^{-1}$ \\
\hline$E_{\mathrm{d}}$ (centre of the Gaussian distribution, donors) & $0.87 \mathrm{eV}$ \\
\hline$E_{\mathrm{a}}$ (centre of the Gaussian distribution, acceptors) & $1.25 \mathrm{eV}$ \\
\hline$w_{\mathrm{d}}=w_{\mathrm{a}}$ (width of the Gaussian distributions) & $0.12 \mathrm{eV}$ \\
\hline$\mu_{\mathrm{n}}$ (free electron mobility) & $10 \mathrm{~cm}^{2} \mathrm{~V}^{-1} \mathrm{~s}^{-1}$ \\
\hline$\mu_{\mathrm{p}}$ (free hole mobility) & $1 \mathrm{~cm}^{2} \mathrm{~V}^{-1} \mathrm{~s}^{-1}$ \\
\hline
\end{tabular}
acceptor states are negatively charged. Once $n_{0}$ and $p_{0}$ are known, $\sigma$ and $L_{a m b}$ are computed from Eqs. (A4) and (A14). After that, the same system of equations is solved for a slightly different generation rate $G+\delta G$, and $\tau^{\prime}$ is calculated by approximating the derivatives in Eq. (9) by the quotient

TABLE I. Parameters used in the numerical simulations. 
of the differentials. It was verified that the numerical calculation always gives $\tau_{n}{ }^{\prime}=\delta n_{T} / \delta G=\tau_{p}{ }^{\prime}=\delta p_{T} / \delta G$.

The calculations were made in a temperature range from $130 \mathrm{~K}$ to $460 \mathrm{~K}$, in $30 \mathrm{~K}$ steps, that is for twelve temperatures. The lower temperature was chosen above the limit beyond which hopping transitions between localized states can be neglected for a-Si: $\mathrm{H}$, while the top temperature was chosen below the limit of structural changes in the material. For each temperature, 13 different generation rates were evaluated, between $5.25 \times 10^{22}$ and $8.81 \times 10^{17} \mathrm{~cm}^{-3} \mathrm{~s}^{-1}$. Each generation rate was obtained dividing the previous one by 2.5 .

For each temperature and generation rate, the parameters $\gamma_{\sigma}$ and $\gamma_{L}$ are obtained from the equations

$$
\begin{gathered}
\gamma_{\sigma}=\frac{\ln \left(\sigma\left(G_{i-1}\right)\right)-\ln \left(\sigma\left(G_{i}\right)\right)}{\ln \left(G_{i-1}\right)-\ln \left(G_{i}\right)}, \\
\gamma_{L}=\frac{\ln \left(L_{a m b}\left(G_{i-1}\right)\right)-\ln \left(L_{a m b}\left(G_{i}\right)\right)}{\ln \left(G_{i-1}\right)-\ln \left(G_{i}\right)},
\end{gathered}
$$

where $G_{i-1}=G_{i} / 2.5$. Expressing $\sigma$ and $L_{a m b}$ as functions of $\gamma_{\sigma}$ and $\gamma_{L}$, respectively, allows obtaining simple expressions for the derivatives with respect to the generation rate. For a given temperature, $\gamma_{\sigma}$ and $\gamma_{L}$ have a weak dependence on the generation rate, so it is possible to obtain them from two adjacent illuminations. For larger differences between generation rates, errors in the calculation of these parameters increase, thus increasing the errors in evaluating the DOS.

In Fig. 1, we plot the originally introduced DOS (line) and the values obtained from the application of Eqs. (4) and (5) (symbols). It can be seen that the points calculated with the higher temperatures and lower generation rate values (lower energies) start to separate from the theoretical curve. For the rest of the points, the agreement is excellent.

Figure 2 compares the values obtained with the pair of Eqs. (1) and (3) and with the pair of Eqs. (4) and (5), for $T=130 \mathrm{~K}$. It can be seen that Eqs. (1) and (3) provide a good approximation to the theoretical curve at low energies for the lower generation rate values, but the error increases at high energies as the generation rate increases. The same

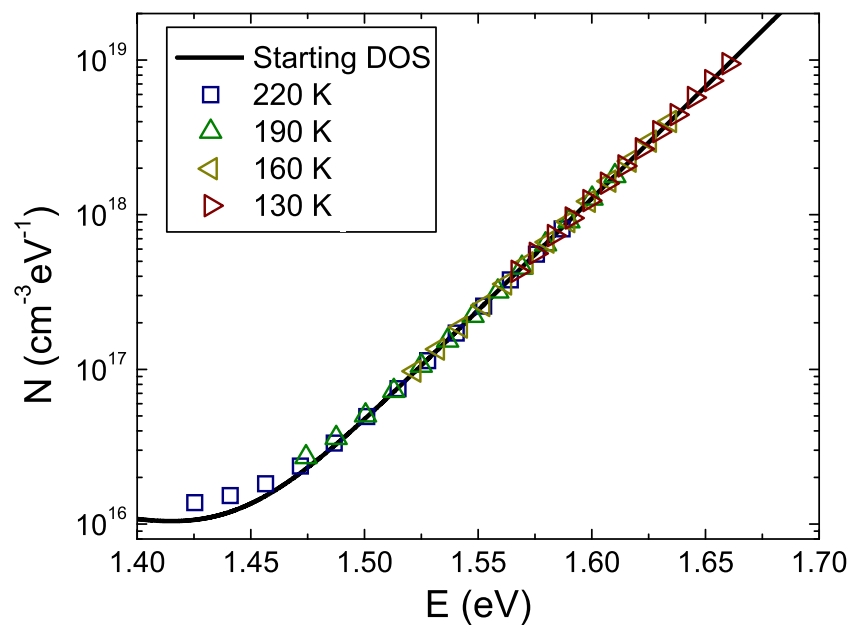

FIG. 1. DOS of Table I (line) and results obtained from the application of Eqs. (4) and (5) (symbols), for the temperatures indicated in the figure and generation rates in the range from $8.81 \times 10^{17}$ to $5.25 \times 10^{22} \mathrm{~cm}^{-3} \mathrm{~s}^{-1}$.

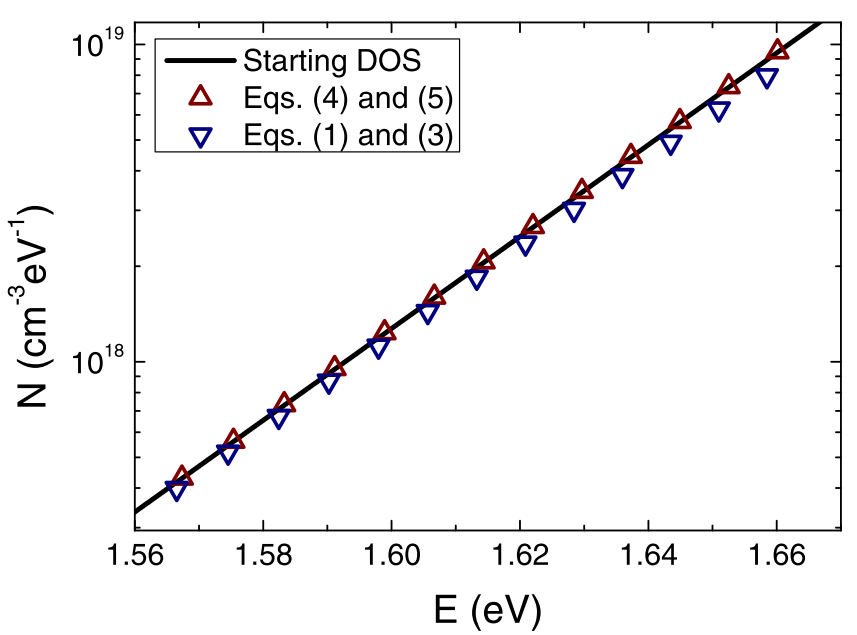

FIG. 2. DOS of Table I (line) and results obtained from the application of Eqs. (4) and (5) (up triangles) and Eqs. (1) and (3) (down triangles), for $\mathrm{T}=130 \mathrm{~K}$ and generation rates in the range from $8.81 \times 10^{17}$ to $5.25 \times 10^{22} \mathrm{~cm}^{-3} \mathrm{~s}^{-1}$.

behavior, but less pronounced, is observed for higher temperatures. Unfortunately, $\tau^{\prime}$ can only be obtained from MGT/ OPG for high values of the generation rate, where the errors of Eqs. (1) and (3) are the largest.

Figure 3 presents the values obtained from Eqs. (7) and (8) for the DOS of Table I. The deepest points corresponding to lower generation rates and higher temperatures deviate from the theoretical curve, but from the other points the VBT can be obtained with excellent accuracy.

One of the advantages of using Eqs. (1) and (3); (4) and (5); or (7) and (8) with a single temperature to get the slope of the band tails is that the error in the determination of $N\left(E_{C, V}\right)$ has no influence on the error for $T_{C, V}$. This can be easily demonstrated by error propagation.

\section{EXPERIMENTAL}

The deduced equations have been tested experimentally on an undoped a-Si:H sample deposited on glass by Plasma Enhanced Chemical Vapor Deposition (PECVD).

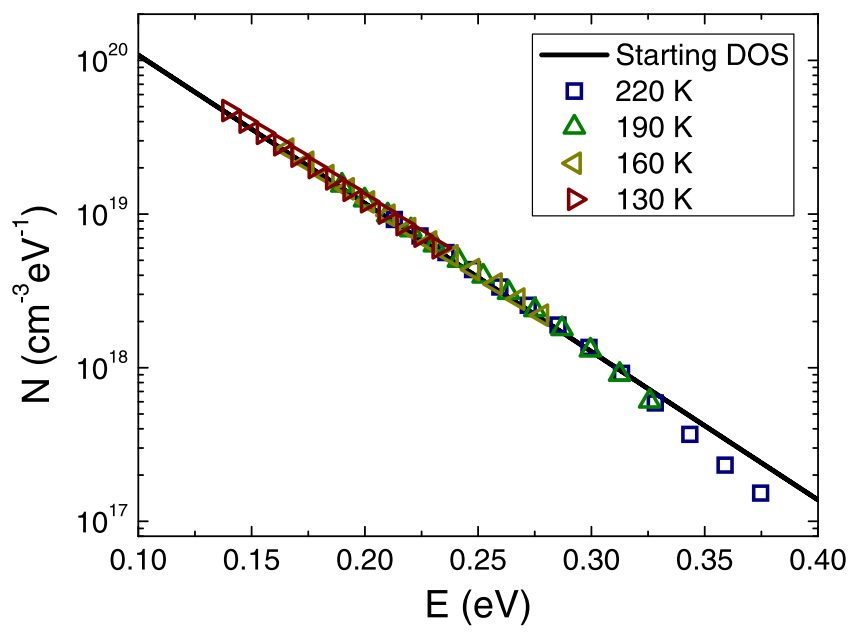

FIG. 3. DOS of Table I (line) and results obtained from the application of Eqs. (7) and (8) (symbols), for the temperatures indicated in the figure and generation rates in the range from $8.81 \times 10^{17}$ to $5.25 \times 10^{22} \mathrm{~cm}^{-3} \mathrm{~s}^{-1}$. 
The deposition was performed in a conventional capacitively coupled reactor operating at $50 \mathrm{MHz}$, from pure silane at a flow rate of $40 \mathrm{sccm}$, a chamber pressure of 0.53 Torr, and a substrate temperature of $220^{\circ} \mathrm{C}$. Silver paint electrodes were deposited on top of the sample for electrical contacts, and disposed in a coplanar geometry with a separation of $1 \mathrm{~mm}$ between them. The ohmicity of the contacts was checked. The sample was light-soaked during $2 \mathrm{~h}$ with a He-Ne laser $\left(100 \mathrm{~mW} / \mathrm{cm}^{2}\right)$ to minimize the effects of light-induced changes on the transport parameters during the experiments.

First, we made some measurements to obtain the basic parameters of the sample. The film thickness was measured using interference techniques from the UV- vis (400-1750 nm) transmittance and reflectance spectra, obtaining a value of $L=1450 \mathrm{~nm}$. The absorption coefficient in the strong absorption region was also evaluated from the UV-vis spectrum. The subgap absorption coefficient was measured by means of the FTPS technique. 7 The DOS of the material was evaluated from conventional techniques, in order to compare with results of the new equations deduced in this work. From the absorption coefficient measured with FTPS, we evaluated the slope of the VBT (Urbach tail) at room temperature $(300 \mathrm{~K})$, and then we corrected to the measured temperature with Stutzmann's relation: ${ }^{20} T_{V}^{2}(T)=T_{V}^{2}(0)+T^{2}$. With the MPC technique we evaluated the CBT, performing measurements from 130 to $190 \mathrm{~K}$ in $20 \mathrm{~K}$ steps.

At a temperature of $175 \mathrm{~K}$, we made the basic measurements needed for the application of the deduced equations: SSPC, SSPG, and MGT. As a light source we used a He-Ne laser $(632.8 \mathrm{~nm})$ and the different fluxes $\Phi$ have been achieved with a neutral filter of variable density. To measure the flux we used a calibrated silicon photodiode. As usual, we assigned to the generation rate a constant value equal to its mean value as a function of depth, neglecting the reflectance at the back surface and assuming a uniform illumination between the contacts

$$
G=\Phi \frac{(1-r)}{L}\left(1-e^{-\alpha L}\right),
$$

where $r$ is the frontal face reflection coefficient.

The SSPC and SSPG measurements were performed at the GeePs Laboratory (Gif-sur-Yvette, France) with an automated equipment described in detail elsewhere. ${ }^{21}$ Samples were kept in vacuum while the temperature was lowered to $175 \mathrm{~K}$. The generation rate was varied between $10^{19}$ and $2.7 \times 10^{21} \mathrm{~cm}^{-3} \mathrm{~s}^{-1}$. We applied a voltage of $20 \mathrm{~V}$ between the electrodes and we measured the photocurrent with an electrometer. From the SSPC measurements we obtained the $\gamma_{\sigma}$ coefficient as a function of generation rate, applying Eq. (28) to two consecutive pairs of values $\left(G_{i}, \sigma_{i}\right)$. From SSPG measurements we evaluated the ambipolar diffusion length $L_{a m b}$ for each value of $G$. A plot of $L_{a m b} v s$. $G$ on logarithmic scales showed an approximately linear behavior; therefore a single value for $\gamma_{L}$ was obtained, $\gamma_{L}=-0.157$. The negative sign implies that $L_{a m b}$ decreases with an increase in $G$, as expected due to the splitting of the quasi-Fermi levels and the increase of the density of recombination states.
The experimental setup for the MGT measurements is shown in Fig. 1 of Ref. 12. A laser light, linearly polarized in the vertical direction, is split into two coherent beams that finally interfere on the position of the sample. Two acoustooptic modulators are used to introduce a small frequency shift $\Delta f$ between the beams, which causes the intensity grating to move with a constant velocity proportional to $\Delta f$. The experimental quantity that is measured with an electrometer is a dc short circuit current, resulting from the movement of the intensity grating. A plot of this current as a function of $\Delta f$ results in a well-defined peak for a certain frequency, which can be used to obtain the small-signal recombination lifetime $\tau^{\prime} .{ }^{12,14}$ At the IFIS-Litoral Laboratory (Santa Fe, Argentina), we have measured $\tau^{\prime}$ for the same temperatures and in the same range of generation rates used for the SSPC and SSPG experiments, obtaining values between $2 \times 10^{-5}$ and $10^{-4} \mathrm{~s}$. Therefore, we have most of the parameters needed to apply Eqs. (4), (5), (7), and (8); except for the capture coefficients, the mobilities, and the values of the DOS at the band edges.

For simplicity, we assumed the same value for the ratio of the capture coefficients in the valence and conduction band tail, $R=R_{C}=R_{V}$. The capture coefficients, the mobilities, and the DOS at the band edges have been obtained by minimizing simultaneously the error between the points calculated with Eqs. (4) and (5) and the CBT measured by MPC, and the points found with Eqs. (7) and (8) and the VBT obtained from FTPS. The range of variation allowed for the unknown parameters has been taken from the literature: ${ }^{16,22,23} N\left(E_{C}\right), N\left(E_{V}\right) \in\left[1 \times 10^{21}, 9 \times 10^{21}\right] \mathrm{cm}^{-3} \mathrm{eV}^{-1}$, $R \in[0.1,10], \mu_{n} \in[5,20] \mathrm{cm}^{2} \mathrm{~V}^{-1} \mathrm{~s}^{-1}$, and $\mu_{p} \in[0.3,5] \mathrm{cm}^{2}$ $\mathrm{V}^{-1} \mathrm{~s}^{-1}$. In Table II, we present the values of the parameters that minimize these errors. The parameters found correspond to those expected for a good quality material.

\section{RESULTS AND DISCUSSION}

We have found that, in Eq. (4), $\tau^{\prime} \gg \frac{\gamma_{\sigma} \sigma}{q \mu_{n} G}$, while in Eq. (7) $\tau^{\prime} \gg\left(\frac{1}{2}+\gamma_{L}\right) \frac{L_{a m b}^{2}}{k_{b} T \mu_{p}}$, so these terms can actually be neglected. This occurs because in a-Si:H at low temperatures the trapped charge is much larger than the free charge.

In Fig. 4, we present the experimental results obtained for the CBT region. The dashed lines correspond to MPC measurements for different temperatures, while the thick line is the upper envelope of the individual frequency scans

TABLE II. Material parameters obtained for the sample of a-Si:H.

\begin{tabular}{lc}
\hline \hline Parameters & Value \\
\hline$T_{\mathrm{V}}$ (characteristic temperature of the VBT at $\left.175 \mathrm{~K}\right)$ & $524 \mathrm{~K}$ \\
$T_{\mathrm{C}}$ (characteristic temperature of the CBT) & $337 \mathrm{~K}$ \\
$N\left(E_{\mathrm{C}}\right)$ (DOS at the conduction band edge) & $9 \times 10^{21} \mathrm{~cm}^{-3} \mathrm{eV}^{-1}$ \\
$N\left(E_{\mathrm{V}}\right)$ (DOS at the valence band edge) & $6.5 \times 10^{21} \mathrm{~cm}^{-3} \mathrm{eV}^{-1}$ \\
$c_{\mathrm{n}}$ (electron capture coefficient) & $1.4 \times 10^{-9} \mathrm{~cm}^{3} \mathrm{~s}^{-1}$ \\
$c_{\mathrm{p}}$ (hole capture coefficient) & $1.8 \times 10^{-9} \mathrm{~cm}^{3} \mathrm{~s}^{-1}$ \\
$\mu_{\mathrm{n}}$ (free electron mobility) & $6.4 \mathrm{~cm}^{2} \mathrm{~V}^{-1} \mathrm{~s}^{-1}$ \\
$\mu_{\mathrm{p}}$ (free hole mobility) & $0.32 \mathrm{~cm}^{2} \mathrm{~V}^{-1} \mathrm{~s}^{-1}$ \\
\hline \hline
\end{tabular}




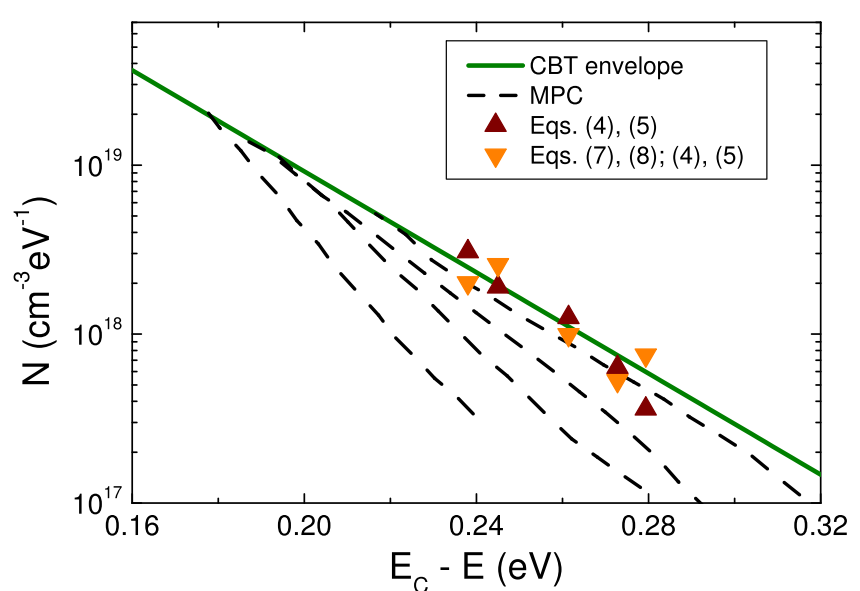

FIG. 4. The thick line is the CBT found through the envelope of the individual MPC results (in dashed lines). The up triangles are the points acquired with Eqs. (4) and (5), while the down triangles are the points found using Eqs. (4), (5), (7), (8), and the VBT parameters.

performed at different temperatures, which reproduces the actual DOS as demonstrated in Ref. 9. The five up triangle points of Fig. 4 correspond to the application of Eqs. (4) and (5), with the values of $\tau^{\prime}$ measured at temperatures of $176 \pm 3 \mathrm{~K}$ and generation rates in the interval $\left[1.6 \times 10^{20}\right.$, $\left.2.7 \times 10^{21}\right] \mathrm{cm}^{-3} \mathrm{~s}^{-1}$. To obtain the five down triangle symbols of Fig. 4, we first used Eqs. (7) and (8) with $T_{V}$ (found from the Urbach tail and Stutzmann's formula) to get $\tau^{\prime}$, and then again Eqs. (4) and (5) to get the CBT points. As can be seen, there is a good correspondence between the CBT deduced from MPC and the points obtained from the application of our formulas. If we concentrate on the up triangles, the four points of higher energy (corresponding to higher generation rates) follow the MPC envelope more closely than the fifth point of lower energy. This is probably due to an error in the determination of $\tau^{\prime}$. It is known from theoretical considerations that MGT underestimates $\tau^{\prime}$ for low generation rates, when the sample is not in the lifetime regime. ${ }^{12}$ As can be seen in Eq. (4), an underestimation of $\tau^{\prime}$ would produce an underestimation of the DOS. On the other hand, the down triangles show that a measurement of the VBT can be used to reproduce the CBT with good accuracy, and what is more, the error does not seem to depend on the generation rate.

In Fig. 5, we show the results found for the VBT, where the line corresponds to the DOS found from FTPS measurements. The up triangles have been obtained from Eqs. (7) and (8) and the measured values of $\tau^{\prime}, \sigma, \gamma_{\sigma}, L_{a m b}$, and $\gamma_{L}$, for the same temperatures and generation rates of Fig. 4 . The agreement with the FTPS curve is quite good, although the points show a bit more dispersion than in Fig. 4. Once again we see that the point closer to midgap (corresponding to a lower generation rate) departs from the VBT measured by FTPS, as a consequence of an error in the determination of $\tau^{\prime}$. The down triangles of Fig. 5 are obtained by using the CBT parameters found from MPC and Eqs. (4) and (5) to get $\tau^{\prime}$, and then using Eqs. (7) and (8) to get the VBT points. The agreement with the reference DOS is very good, showing that the VBT can be obtained with good precision if the CBT is measured by MPC.

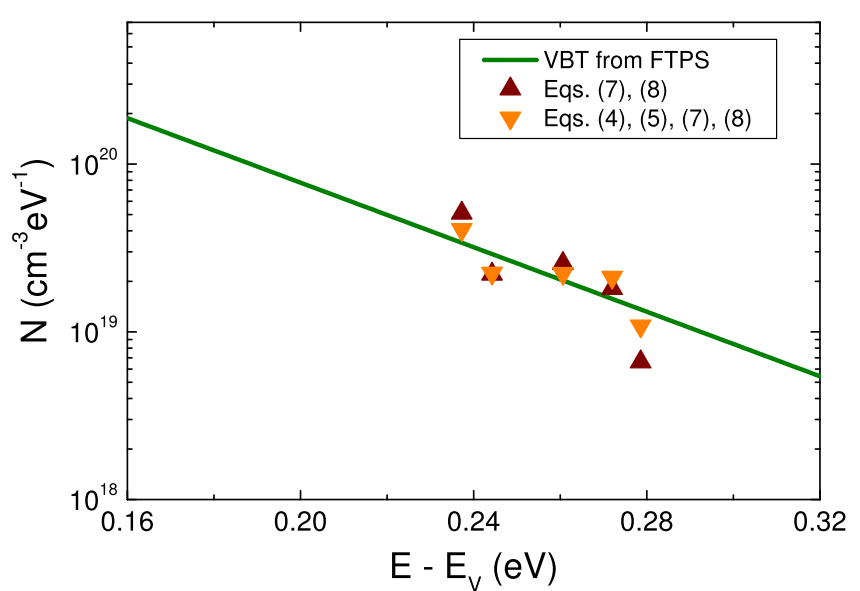

FIG. 5. Valence band tail obtained through FTPS (line) and points found with Eqs. (7) and (8) (up triangles) or using Eqs. (4), (5), (7), (8), and the CBT parameters (down triangles).

It is a mathematical fact that the subtraction of two quantities of nearly the same value amplifies notably the errors of the original quantities. Consequently, a formula proposed for material characterization must avoid this feature as much as possible. We can see this problem in Eqs. (4) and (7), because to calculate the parameters $\gamma_{\sigma}$ and $\gamma_{L}$ we must use the incremental quotients (28) and (29). In Eq. (7), we observe the same problem once more. Inside the denominator's parenthesis the first term is negative (for $T<T_{V}$ ) while the second is positive, and they are of the same order of magnitude for a-Si: $\mathrm{H}$, as can be seen clearly from numerical simulation. Inside the denominator's parenthesis of Eq. (4), we observe another subtraction but in this case luckily the magnitude of the first term is notably bigger than the second one for a-Si:H. Another asymmetry between Eqs. (4) and (7) comes from the value of $L_{a m b}$, whose errors affect more strongly Eq. (7) than Eq. (4) because it appears as a factor squared in the first one. Actually, when we take $L_{a m b} \rightarrow 0$ we see that Eqs. (4) and (5) tend to the well-known Eqs. (1) and (3), which are still a good approximation for the CBT. After analyzing the sources of errors, the smaller dispersion in the points obtained with Eqs. (4) and (5) compared with those found with Eqs. (7) and (8), appears as an expected result.

\section{CONCLUSION}

In this work, we have presented new formulas to obtain the density of states in the band tails of hydrogenated amorphous silicon from photoconductivity-based measurements. First we have deduced the new formulas and tested their accuracy by numerical simulations using typical a-Si:H parameters. Next, we have characterized an a-Si:H sample using well-known methods (FTPS and MPC techniques), and we have made all the necessary measurements for the application of the deduced equations: SSPC to get $\sigma$ and $\gamma_{\sigma}$, SSPG to get $L_{a m b}$ and $\gamma_{L}$, and MGT to get $\tau^{\prime}$. These measurements were performed at a temperature of $\sim 175 \mathrm{~K}$ and for a range of generation rates.

The results obtained from the application of our new formulas are in good agreement with those found with the traditional methods for both band tails, although experimental 
errors affect more strongly Eq. (7) than Eq. (4). We have shown that MGT/OPG measurement to get $\tau^{\prime}$ can be avoided if one of the band tails is measured by one of the traditional methods, since the known band tail can be used in combination with our formulas to get $\tau^{\prime}$. The main disadvantage of the above-derived equations is that they require prior knowledge of the capture coefficients and mobilities of electrons and holes. However, they also open a possibility to estimate these parameters from simple photoconductivity-based measurements.

\section{ACKNOWLEDGMENTS}

We acknowledge support from the CONICET under Project Nos. PUE 22920160100054CO and PIP 0414, the ANPCyT under Project No. PICT 2013-2098, the Universidad Nacional del Litoral under project CAI + D PI 84-099, and the MINCyT - Ecos Sud under Project No. A13E02.

\section{APPENDIX: BASIC EQUATIONS}

The charge neutrality equation for the semiconductor in dark equilibrium is

$$
n_{d k}+\int_{E_{v}}^{E_{c}} \frac{N^{A C C}(E) d E}{1+e^{\left(E-E_{f}\right) / k_{b} T}}-p_{d k}-\int_{E_{v}}^{E_{c}} \frac{N^{D O N}(E) d E}{1+e^{\left(E_{f}-E\right) / k_{b} T}}=0
$$

where $n_{d k}$ and $p_{d k}$ represent the concentrations of electrons and holes in the bands, given by

$$
\begin{gathered}
n_{d k}=N_{C} e^{-\left(E_{C}-E_{f}\right) / k_{b} T}, \\
p_{d k}=N_{V} e^{\left(E_{V}-E_{f}\right) / k_{b} T} .
\end{gathered}
$$

The others terms in Eq. (A1) stand for the concentration of trapped carriers as occupied acceptors and unoccupied donors.

The electrical conductivity $\sigma$ for a given uniform illumination is given by

$$
\sigma=q\left(\mu_{n} n_{0}+\mu_{p} p_{0}\right)
$$

where $n_{0}$ and $p_{0}$ are the free carrier concentrations. If $n_{0} \gg p_{0}$ and $\mu_{n}>\mu_{p}$, as in a-Si:H, we have

$$
\sigma \cong q \mu_{n} n_{0}
$$

The occupation functions for a state with capture coefficients $c_{n}$ and $c_{p}$, and emission coefficients $e_{n}$ and $e_{p}$ are

$$
\begin{gathered}
f_{0}=\frac{c_{n} n_{0}+e_{p}(E)}{c_{n} n_{0}+c_{p} p_{0}+e_{n}(E)+e_{p}(E)}, \\
\left(1-f_{0}\right)=\frac{c_{p} p_{0}+e_{n}(E)}{c_{n} n_{0}+c_{p} p_{0}+e_{n}(E)+e_{p}(E)} .
\end{gathered}
$$

The emission coefficients are defined by

$$
\begin{aligned}
& e_{n}(E)=c_{n} N_{C} e^{\frac{E-E_{C}}{k_{b} T}}, \\
& e_{p}(E)=c_{p} N_{V} e^{\frac{E_{V}-E}{k_{b} T}} .
\end{aligned}
$$

The free lifetimes of electrons and holes are defined by

$$
\begin{gathered}
\tau_{n}=\frac{\left(n_{0}-n_{d k}\right)}{G}, \\
\tau_{p}=\frac{\left(p_{0}-p_{d k}\right)}{G} .
\end{gathered}
$$

At low temperatures and high generation rates, they can be approximated by

$$
\begin{aligned}
\tau_{n} & \cong \frac{n_{0}}{G} \\
\tau_{p} & \cong \frac{p_{0}}{G} .
\end{aligned}
$$

The ambipolar diffusion length is defined by

$$
L_{a m b}=\sqrt{2 k_{b} T \frac{\mu_{n} \tau_{n} \mu_{p} \tau_{p}}{\mu_{n} \tau_{n}+\mu_{p} \tau_{p}}} .
$$

For a semiconductor like undoped a-Si:H we have $\mu_{n} \tau_{n} \gg \mu_{p} \tau_{p}$, so Eq. (A14) can be simplified into

$$
L_{a m b} \cong \sqrt{2 k_{b} T \mu_{p} \tau_{p}}
$$

The concentrations of electrons and holes trapped in gap states are given by

$$
\begin{gathered}
n_{t}=\int_{E_{v}}^{E_{c}} f_{0} N^{A C C}(E) d E, \\
p_{t}=\int_{E_{v}}^{E_{c}}\left[1-f_{0}\right] N^{D O N}(E) d E .
\end{gathered}
$$

The quasi-Fermi levels for trapped carriers are defined as

$$
\begin{aligned}
& E_{t p}=E_{V}+k_{b} T \ln \left(\frac{c_{p} N_{V}}{c_{n} n_{0}+c_{p} p_{0}}\right), \\
& E_{t n}=E_{C}-k_{b} T \ln \left(\frac{c_{n} N_{C}}{c_{n} n_{0}+c_{p} p_{0}}\right),
\end{aligned}
$$

and the effective densities of states at the band edges are

$$
\begin{aligned}
& N_{C}=\int_{E_{C}}^{+\infty} \frac{N(E) d E}{1+e^{\frac{\left(E-E_{f}\right)}{k_{b} T}}}, \\
& N_{V}=\int_{-\infty}^{E_{V}} \frac{N(E) d E}{1+e^{\frac{\left(E_{f}-E\right)}{k_{b} T}}} .
\end{aligned}
$$

As a consequence of the charge neutrality equation, the recombination lifetime $\tau_{R}$ is equivalently defined as

$$
\tau_{R}=\frac{n_{T}}{G}=\frac{p_{T}}{G} .
$$

\footnotetext{
${ }^{1}$ M. Vanecek, J. Kocka, J. Stuchlık, Z. Kozısek, O. Stika, and A. Trıska, Sol. Energy Mater. 8, 411 (1983).

${ }^{2}$ P. Jensen, Solid State Commun. 76, 1301 (1990).

${ }^{3}$ J. A. Schmidt, R. Arce, R. H. Buitrago, and R. R. Koropecki, Phys. Rev. B $\mathbf{5 5}, 9621$ (1997)
} 
${ }^{4}$ M. Vanecek, J. Kocka, J. Stuchlik, and A. Triska, Solid State Commun. 39, 1199 (1981).

${ }^{5}$ C. R. Wronski, B. Abeles, T. Tiedje, and G. D. Cody, Solid State Commun. 44, 1423 (1982).

${ }^{6}$ W. B. Jackson and N. M. Amer, Phys. Rev. B 25, 5559 (1982).

${ }^{7}$ M. Vanecek and A. Poruba, Appl. Phys. Lett. 80, 719 (2002).

${ }^{8}$ H. Oheda, J. Appl. Phys. 52, 6693 (1981).

${ }^{9}$ C. Longeaud and J. P. Kleider, Phys. Rev. B 45, 11672 (1992).

${ }^{10}$ J. A. Schmidt and C. Longeaud, Appl. Phys. Lett. 85, 4412 (2004).

${ }^{11}$ F. Ventosinos, N. Budini, C. Longeaud, and J. A. Schmidt, J. Phys. D: Appl. Phys. 44, 295103 (2011)

${ }^{12}$ F. Ventosinos, C. Longeaud, and J. A. Schmidt, J. Non-Cryst. Solids 358, 2031 (2012).

${ }^{13}$ C. Longeaud and J. A. Schmidt, J. Non-Cryst. Solids 358, 2052 (2012).

${ }^{14}$ C. Longeaud, F. Ventosinos, and J. A. Schmidt, J. Appl. Phys. 112, 023709 (2012).
${ }^{15}$ D. Ritter, E. Zeldov, and K. Weiser, Appl. Phys. Lett. 49, 791 (1986).

${ }^{16}$ R. A. Street, Hydrogenated Amorphous Silicon (Cambridge University Press, Cambridge, 1991).

${ }^{17}$ S. K. O’Leary, S. R. Johnson, and P. K. Lim, J. Appl. Phys. 82, 3334 (1997).

${ }^{18}$ J. Furlan, F. Smole, and P. Popovic, in Amorphous Silicon Technology, edited by E. A. Schiff, M. J. Thompson, A. Madan, K. Tanaka, and P. G. LeComber (Mater. Res. Soc. Symp. Proc., 1993), Vol. 297, pp. 363-368.

${ }^{19}$ V. Halpern, Philos. Mag. B 54(6), 473-482 (1986).

${ }^{20}$ M. Stutzmann, Philos. Mag. Lett. 66, 147 (1992).

${ }^{21}$ A. Fath Allah, F. Ventosinos, and C. Longeaud, J. Phys.: Conf. Ser. 558, 012011 (2014).

${ }^{22}$ T. Searle, Properties of Amorphous Silicon and Its Alloys (INSPEC, London, 1998), p. 119.

${ }^{23}$ T. Tiedje, J. M. Cebulka, D. L. Morel, and B. Abeles, Phys. Rev. Lett. 46, 1425 (1981). 